\title{
Proliferaciones Oncocíticas de Glándulas Salivales: Estudio Estructural e Inmunohistoquímico de 7 Casos
}

\author{
Oncocytic Proliferations of Salivary Glands: Structural \\ and Immunohistochemical Study of 7 Cases
}

\author{
Rodolfo Esteban Avila1; María Elena Samar²; Ismael Bernardo Fonseca'; \\ Alberto Gustavo Corball ${ }^{3}$; Víctor Carriel ${ }^{4}$; Laura García-Martinez ${ }^{4}$, Ismael Rodríguez ${ }^{2}$
}

\begin{abstract}
AVILA, R. E.; SAMAR, M. E.; FONSECA, I. B.; CORBALL, A. G.; CARRIEL, V.; GARCÍA-MARTINEZ, L. \& RODRÍGUEZ, I. Proliferaciones oncocíticas de glándulas salivales: estudio estructural e inmunohistoquímico de 7 casos. Int. J. Odontostomat., 13(1):82-88, 2019.
\end{abstract}

RESUMEN: Los oncocitos son células originadas probablemente por transformación metaplásica del epitelio ductal o acinar de parótida y submandibular. Su proliferación puede originar condiciones patológicas que incluyen hiperplasias oncocíticas adenomatosas multinodulares (HOAM), oncocitomas y carcinomas oncocíticos. Los tumores oncocíticos constituyen el $1 \%$ de todos los tumores salivales y entre el 82 y $90 \%$ se desarrollan en la parótida; el resto se divide entre la glándula submandibular y las glándulas salivales menores. Las hiperplasias oncocíticas multinodulares son extremadamente raras. En el presente trabajo se analizaron 5 casos de oncocitomas de parótida y dos casos de HOAM, uno de parótida y otro de submandibular y se describieron las características estructurales e inmunohistoquímicas de los oncocitos. Cortes seriados de las biopsias incluidas en parafina se colorearon con Hematoxilina - Eosina, Hematoxilina/ácido fosfotúngstico (PTA/H), PAS y se marcaron con AC antimitocondrial, CK 5/6, CK 20 y EMA. Los tumores mostraron un crecimiento nodular encapsulado por tejido conectivo denso. En los cortes histológicos se identificaron oncocitos eosinófilos (oscuros) y granulaciones violáceas con PTA/H. En dos casos de parótida y el caso de HOAM de submandibular presentaron además oncocitos claros PAS positivos. La inmunomarcación fue positiva en todas las células siendo la marcación para mitocondrias periférica en los oncocitos claros. Las células eosinofílicas PTA/H positivas y con fuerte marcación con AC antimitocondrial, CKs y EMA confirman el diagnóstico de patología oncocítica. En tres casos coexisten oncocitos claros y oscuros. Las células claras son oncocitos que acumulan glucógeno en su citoplasma desplazando a las mitocondrias hacia la periferia. En el diagnóstico diferencial de este tumor debemos considerar los tumores salivales con células claras, el carcinoma renal metastásico, el tumor de Whartin, la variante de células claras del carcinoma epitelial/mioepitelial y el carcinoma mucoepidermoide con metaplasia oncocítica.

PALABRAS CLAVE: glándulas salivales; oncocitos; oncocitoma; hiperplasia oncocítica adenomatosa multinodular; histología; inmunohistoquímica.

\section{INTRODUCCION}

Mandel \& Carrao (2005) describen al oncocito como una célula que se origina probablemente a partir de la transformación metaplásica del epitelio ductal o acinar de las glándulas parótida y submandibular.

Se ha sugerido que un defecto metabólico intracelular asociado a una mitocondriopatía dependiente de la edad origina esa metaplasia. Por el con- trario, estudios ultraestructurales indican que los oncocitos salivales se originarían como consecuencia de una hiperplasia celular adaptativa compensatoria por una mutación somática (Zagólski et al., 2006; Kumar et al., 2017).

En glándulas salivales normales estas células aparecen esporádicamente y con mayor frecuencia en

\footnotetext{
${ }^{1}$ Cátedra de Biología Celular, Histología y Embriología. Facultad de Ciencias Médicas. Universidad Nacional de Córdoba, Córdoba, Argentina. ${ }^{2}$ Departamento de Biología Bucal. Facultad de Odontología. Universidad Nacional de Córdoba, Córdoba, Argentina.

${ }^{3}$ Fundación para la Educación, Investigación y Prevención en Cabeza y Cuello. Córdoba. Argentina.

${ }^{4}$ Grupo Ingeniería Tisular, Universidad de Granada, Granada, España.

Subsidio 05/H575. Secretaria de Ciencia y Tecnología, Universidad Nacional de Córdoba, Argentina.
} 
individuos de edad avanzada (Politi et al., 2005). Se reconocen con la tinción de H\&E; presentan abundantes mitocondrias que le dan al citoplasma un aspecto granuloso eosinofílico. Sin embargo, como bien relatan Vera-Sampere \& Vera-Sirera (2011), otros tipos celulares tienen una apariencia oncocítica que obliga a la aplicación de la inmunohistoquímica para su correcta identificación. Los anticuerpos antimitocondriales son eficaces para la diferenciación de estas células que, además, son citoqueratinas y EMA positivas (Zhou \& Gao, 2009).

La proliferación de los oncocitos salivales puede originar condiciones patológicas que incluyen hiperplasias oncocíticas multinodulares (HOAM), oncocitomas y carcinomas oncocíticos (Ellis \& Auclair, 2008). Algunos autores consideran que el oncocitoma es la contraparte neoplásica de la HOAM (Loreti et al., 2002).

Los tumores oncocíticos constituyen el $1 \%$ de todos los tumores salivales y entre el 82 y $90 \%$ se desarrollan en la glándula parótida; el resto de los tumores se divide entre la glándula submandibular y las glándulas salivales menores. Las hiperplasias oncocíticas multinodulares son extremadamente raras (Politi et al.; Prabakaran et al., 2010).

Se desarrollan más comúnmente entre la $6^{\circ}$ y $8^{\circ}$ décadas de la vida siendo la edad media de los pacientes de 58 años (El-Naggar et al., 2017).

En este estudio presentamos 5 casos de oncocitoma de parótida y 2 casos de HOAM localizados en parótida y submandibular. Se determinaron las características tintoriales e inmunohistoquímicas de los oncocitos y se hizo una revisión bibliográfica del tema.

\section{MATERIAL Y MÉTODO}

Se realizó el examen histológico de las piezas quirúrgicas correspondientes a cinco oncocitomas ICD-0-Code 8290/0 - International Classification of Diseases for Oncology- (WHO) y dos hiperplasias oncocíticas adenomatosas multinodulares (HOAM) de glándulas salivales, procesadas según la técnica de inclusión en parafina.

Los cortes histológicos coloreados con H\&E fueron revisados de acuerdo a la última clasificación de la OMS, correspondiente al año 2017 (El-Naggar et al.). Cortes seriados de $4 \mu \mathrm{m}$ de espesor fueron utilizados para PAS y Hematoxilina/ácido fosfotúngstico $(\mathrm{PTA} / \mathrm{H})$ y análisis inmunohistoquímico. Los anticuerpos utilizados fueron CK 5/6, CK 20, EMA y anticuerpo antimitocondrial, (Samar et al., 2004). Como control de la positividad de la inmunomarcación con anticuerpo antimitocondrial se utilizaron cortes de parótida donde el anticuerpo marcó las mitocondrias de los conductos estriados (Fig. 1).

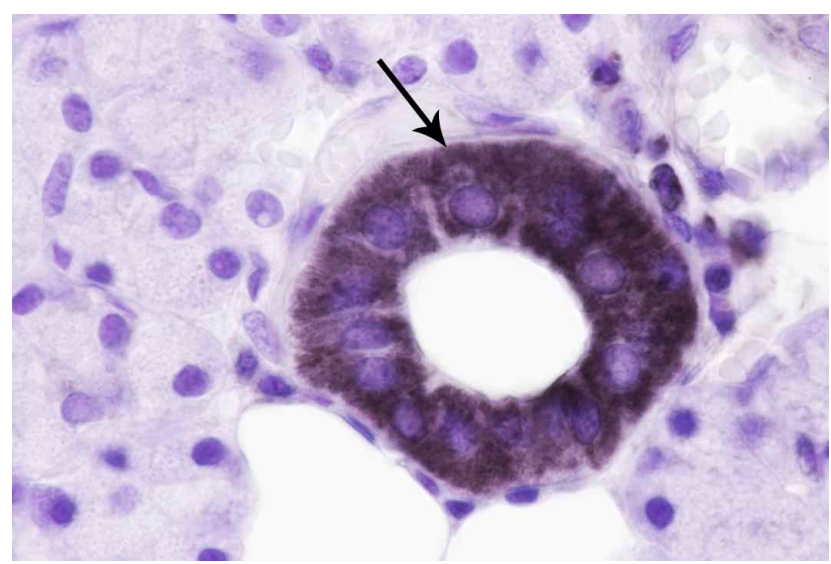

Fig. 1. Corte control. Conducto estriado de glándula parótida (flecha). Anticuerpo antimitocondrial positivo. 400x.

Este trabajo es parte del proyecto "La matriz extracelular y el componente mioepitelial de los tumores epiteliales de glándulas salivales humanas: estudio estructural, histoquímico, inmunohistoquímico y lectinhistoquímico, autorizado por el Comité de Etica del Hospital Nacional de Clínicas. Facultad de Ciencias Médicas. Universidad Nacional de Córdoba, Argentina. Registro 188/14.

\section{RESULTADOS}

En la Tabla I se presentan los casos estudiados y se especifican los datos de edad, sexo, localización y diagnóstico histopatológico mientras que en la Tabla II se sintetizan las características macroscópicas y citológicas de los oncocitomas y HOAM.

En todos los casos los pacientes consultaron por una masa indolora de varios meses de evolución.

Oncocitomas. En el examen histopatológico se observó que todos los oncocitomas desarrollaron un patrón nodular encapsulado. En el caso 3 la cápsula fibrosa estaba infiltrada por oncocitos tumorales lo que indicaba una tendencia de crecimiento más agresivo. 
AVILA, R. E.; SAMAR, M. E.; FONSECA, I. B.; CORBALL, A. G.; CARRIEL, V.; GARCíA-MARTINEZ, L. \& RODRÍGUEZ, I. Proliferaciones oncocíticas de glándulas salivales: estudio estructural e inmunohistoquímico de 7 casos. Int. J. Odontostomat., 13(1):82-88, 2019.

Tabla I. Proliferaciones oncocíticas de glándulas salivales: casuística.

\begin{tabular}{llll}
\hline Diagnóstico & Edad & Sexo & Localización \\
\hline Caso 1: Oncocitoma & 26 años & M & Parótida \\
Caso 2: Oncocitoma & 64 años & M & Parótida \\
Caso 3: Oncocitoma & 55 años & F & Parótida \\
Caso 4: Oncocitoma & 76 años & M & Parótida \\
Caso 5: Oncocitoma & 45 años & F & Parótida \\
Caso 6: HOAM & 50 años & F & Submandibular \\
Caso 7: HOAM & 60 años & F & Parótida \\
\hline
\end{tabular}

M. masculino. F. femenino. HOAM. hiperplasia oncocítica adenomatosa multinodular.

Tabla II. Proliferaciones oncocíticas de glandulas salivales: características macroscópicas y citológicas.

\begin{tabular}{|c|c|c|c|c|}
\hline Caso & Márgenes & Aspecto Macroscópico & Focos Hemorrágicos & Características Citológicas \\
\hline 1 Oncocitoma & Encapsulado & $\begin{array}{l}\text { Nódulo amarillo blanquecino } \\
\text { de consistencia elástica. } \\
\text { Superficie externa irregular }\end{array}$ & Ausentes & Oncocitos oscuros \\
\hline 2 - Oncocitoma & Encapsulado & $\begin{array}{l}\text { Nódulo sólido pardo } \\
\text { amarillento de aspecto firme y } \\
\text { homogéneo. } \\
\text { Superficie externa irregular }\end{array}$ & Ausentes & Oncocitos oscuros \\
\hline 3- Oncocitoma & $\begin{array}{lr}\text { Encapsulado. } \\
\text { Cápsula } & \text { fibrosa } \\
\text { infiltrada en } & \text { zonas } \\
\text { por } & \text { células } \\
\text { neoplásicas } & \end{array}$ & $\begin{array}{l}\text { Lesión blanquecina y de } \\
\text { consistencia blanda con áreas } \\
\text { hemorrágicas. } \\
\text { Superficie externa } \\
\text { polilobulada }\end{array}$ & Presentes & Oncocitos oscuros y claros \\
\hline 4 - Oncocitoma & Encapsulado & $\begin{array}{l}\text { Nódulo color pardo œn áreas } \\
\text { congestivas }\end{array}$ & Presentes & Oncocitos oscuros \\
\hline 5- Oncocitoma & Encapsulado & $\begin{array}{l}\text { Nódulo color pardo } \\
\text { amarillento y consistencia } \\
\text { elástica }\end{array}$ & Ausentes & Oncocitos oscuros \\
\hline 6 - HOAM & Sin cápsula & $\begin{array}{l}\text { Lesión multinodular sólida de } \\
\text { color grisáceo y consistencia } \\
\text { firme }\end{array}$ & $\begin{array}{l}\text { Presentes } \\
\text { con depósitos de } \\
\text { hemosiderina }\end{array}$ & Oncocitos oscuros y claros \\
\hline 7- HOAM & Límite fibroso fino & $\begin{array}{l}\text { Lesión multinodular sólida, } \\
\text { blanquecina }\end{array}$ & Ausentes & $\begin{array}{l}\text { Oncocitos } \\
\text { claros }\end{array}$ \\
\hline
\end{tabular}

Los cortes histológicos coloreados con H\&E mostraron típicos oncocitos oscuros con citoplasma granular eosinofílico y granulaciones violáceas con PTA/H en cuatro casos de oncocitoma (Fig. 2A). En el caso 3 se observaron además células claras (Fig. 2B) positivas con $\mathrm{PTA} / \mathrm{H}$ y PAS reactivas. Las células se disponían en nidos sólidos o trabéculas y formaban en algunas zonas del tumor estructuras tubulares y seudoductales, algunas con dilatación quística y focos de hemosiderina en su interior. El estroma era escaso y fibroso. En la periferia de las lesiones el tejido glandular era normal, separado por tejido conectivo.

\section{Hiperplasias oncocíticas adenomatosas} multinodulares (HOAM). Las HOAM presentaron un patrón multinodular con tejido conectivo fibroso que separaba cada nódulo. En los cortes histológicos coloreados con H\&E se observaron nódulos de diferente tamaño con una proliferación de oncocitos oscuros con cito- plasma eosinófilo y oncocitos claros con citoplasma vacuolado (Fig. 3) y algunos núcleos con ligera aniso y macrocariosis. Con técnicas especiales (PTA/H y PAS) se demostró que las células claras tenían glucógeno y mitocondrias en su citoplasma y las células oscuras eosinófilas mitocondrias. Focos de hemorragia con depósitos de hemosiderina se encontraron en el caso 6 . El estroma era escaso y fibroso. En la periferia de las lesiones el tejido glandular era normal. En el caso 6 no se observó cápsula limitante en tanto que en el caso 7 una formación fibrosa delgada separaba los nódulos del parénquima normal.

En los siete casos descritos los oncocitos fueron reactivos con anticuerpo antimitocondrial, EMA y CK 5/6 y CK 20 (Figs. 4A,B y 5A,B). Los oncocitos claros eran inmunorreactivos al anticuerpo antimitocondrial en la región periférica del citoplasma. No se identificaron figuras de mitosis ni atipía celular. 

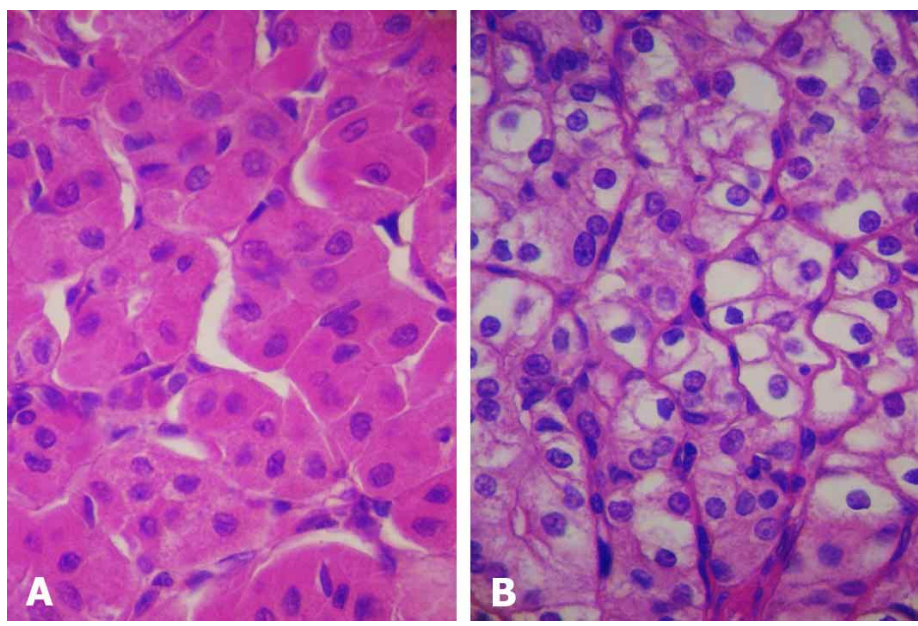

Fig. 2. Oncocitoma A-Oncocitos oscuros. B. Oncocitos claros. H\&E. 400x.

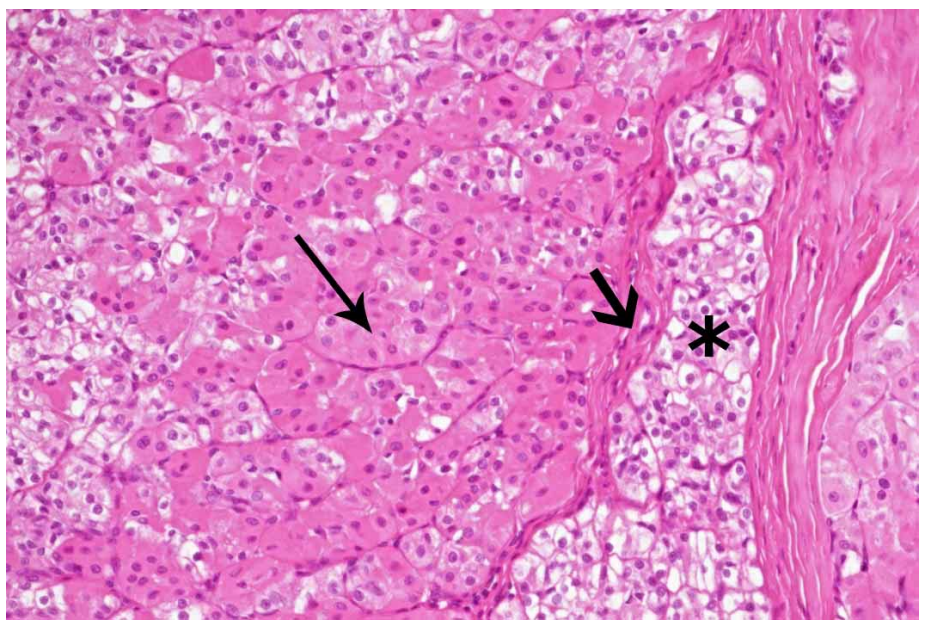

Fig. 3. Hiperplasia oncocitica adenomatosa multinodular. Oncocitos oscuros (flecha fina) y claros (asterisco) en un patrón multinodular separado por tejido conectivo (flecha gruesa). H\&E. 200x.
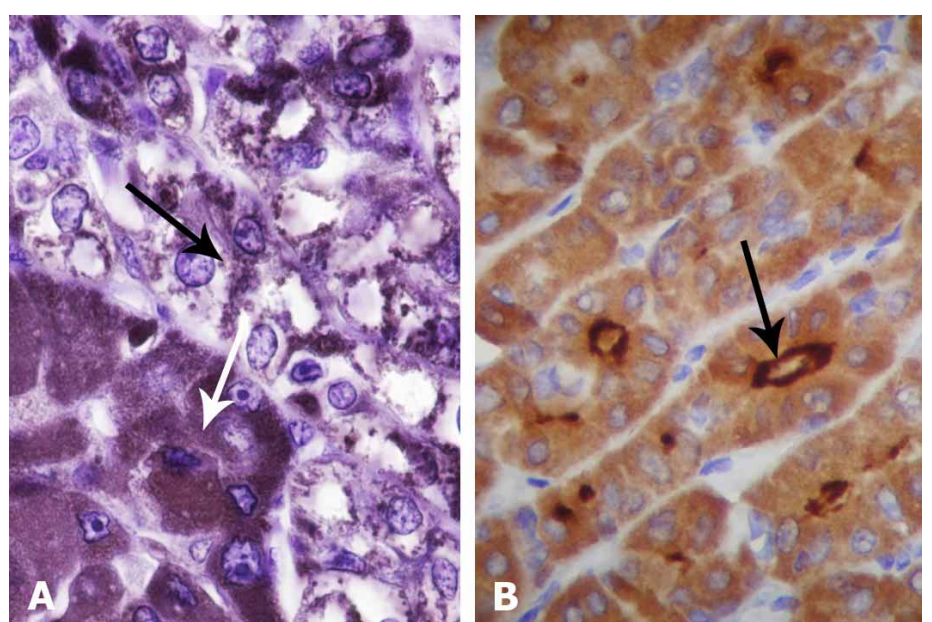

Fig. 4. Oncocitos. Inmunohistoquímica. A-Oncocitos claros (flecha negra) y oscuros (flecha blanca). Anticuerpo antimitocondrial. 400X. B-Oncocitos con inmunomarcación positiva (flecha) para antígeno epitelial de membrana (EMA). 400x.

\section{DISCUSIÓN}

Las lesiones oncocíticas de las glándulas salivales fueron descritas por primera vez por Schaffer en el año 1897. Estas lesiones también se desarrollan en otros órganos como riñón, glándula lagrimal, páncreas, tiroides e hipófisis (Evren et al., 2015).

Las neoplasias oncocíticas comprenden un grupo raro de tumores de localización preferencialmente parotídea correspondiendo aproximadamente al $1 \%$ de todos los tumores de esta glándula (Vlachaki et al., 2009). De acuerdo a la clasificación histológica de los tumores de glándulas salivales de la Organización Mundial de la Salud se diferencian dos categorías: oncocitoma y carcinoma oncocítico (WHO).

Por el contrario, la OMS considera que la oncocitosis es la acumulación y proliferación no neoplásica de oncocitos en un patrón multinodular (HOAM), que compromete casi todo el parénquima glandular; es extremadamente rara y comprende aproximadamente un $0,1 \%$ de las lesiones salivales (Kinoshita el al., 2014; WHO).

Tanto los oncocitomas como las HOAM involucran generalmente a la glándula parótida. No obstante, se describen otras localizaciones menos frecuentes como las glándulas palatinas y submandibular y la región retromolar (Thompson et al., 1996; Palakshappa et al., 2014; Motallebnejad et al., 2015). En nuestra casuística un caso de HOAM se localizó en glándula submandibular.

El oncocitoma se describe como una masa bien circunscripta de oncocitos, rodeada al menos parcialmente por una cápsula fibrosa y de mayor tamaño que otros nódulos oncocíticos de las glándulas salivales (Ellis \& Auclair). Ocurre más comúnmente entre la $6^{\circ}$ y $8^{\circ}$ décadas de la vida (Sepúlveda et al., 2014). Si bien son tumores que aparecen en adultos mayores, en nuestra casuística encontramos oncocitomas en 4 pacientes con edades comprendidas entre los 26 y 55 años. 


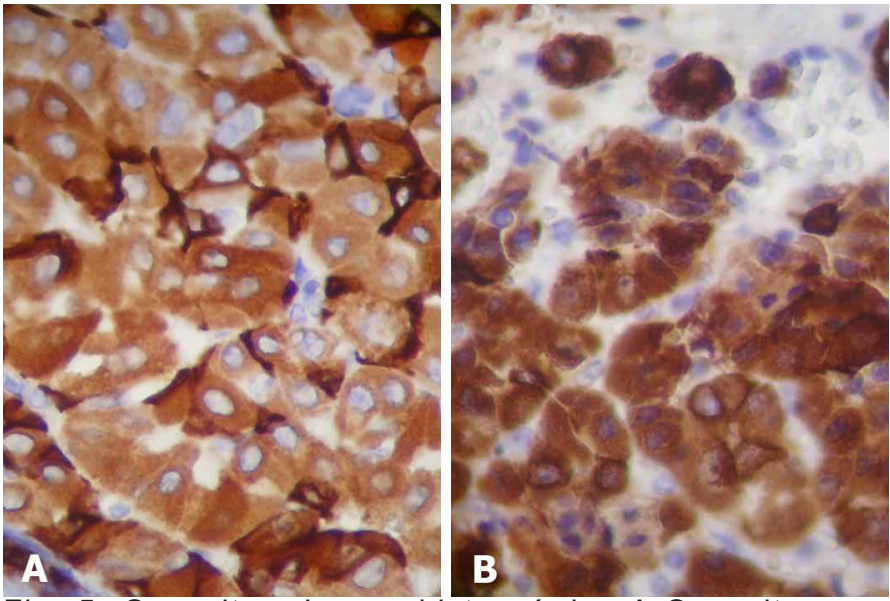

Fig. 5. Oncocitos. Inmunohistoquímica A-Oncocitos con inmunomarcación positiva para CK 5/6.400x. B-Oncocitos con inmunomarcación positiva para CK 20. 400x

En cuanto a las HOAM, se desarrollan principalmente en mujeres en la $6^{\circ}$ década de la vida (Politi et al.), lo que coincide con los casos descritos en el presente trabajo. Son lesiones benignas no neoplásicas con nódulos de tamaño variable. Su diferenciación con los oncocitomas puede resultar oscura y problemática. Generalmente se considera que el oncocitoma es una lesión solitaria bien definida mientras que la HOAM es multifocal y no altera la arquitectura del parénquima normal (Çiris et al., 2011). Un criterio empleado para su diferenciación con los oncocitomas suele ser su encapsulación mínima; además, en los oncocitomas no se identifica parénquima glandular sano en el interior de la lesión (Alcaraz-Mateos et al., 2012). No obstante, hay oncocitomas desprovistos de cápsula fibrosa. Si bien las diferencias histológicas, moleculares, inmunohistoquímicas y citogenéticas no están claramente definidas, éstas no resultan importantes para su pronóstico y tratamiento (Ellis \& Auclair). Su tratamiento es quirúrgico observándose recurrencias en casos de resección incompleta de la lesión. En la literatura no se han informado casos de transformación maligna ni metástasis (Strassburger et al., 1999; Kontaxis et al., 2004; Rooper et al., 2017).

Se ha sugerido que los oncocitomas se originan de las células de los conductos intercalados. Y, como mencionan Zhou \& Gao, esto es consistente con la observación de que la mayoría de los oncocitomas se localizan en la glándula parótida donde estos conductos son abundantes. Las pruebas inmunohistoquímicas positivas (CK y EMA) confirman su origen en los conductos intercalados. En todos los casos analizados en este estudio observamos marcación positiva de los oncocitos con CK 5/6, CK 20 y EMA además de la fuerte positividad con PTA/H y anticuerpo mitocondrial.
Por otro lado, si bien se considera que los oncocitos contienen un citoplasma abundante con apetencia tintorial por la eosina, también se describen en la literatura células con citoplasma claro (Saqi et al., 2007; Palakshappa et al.).

El cambio claro de los oncocitos se produce en pequeños grupos celulares o corresponde al tipo celular principal del tumor o de la oncocitosis. Estos oncocitos claros son PAS positivos debido a la acumulación de glucógeno en su citoplasma con desplazamiento de las mitocondrias hacia la periferia celular (Saqi et al.; Prabakaram et al.). Con $\mathrm{H} \& \mathrm{E}$ se aprecian los oncocitos "oscuros" eosinofílicos dispuestos entre los oncocitos "claros", como observamos en los casos 3, 6 y 7 .

En el diagnóstico diferencial de estas patologías se incluyen los carcinomas oncocíticos y los tumores salivales con células claras, el carcinoma renal metastásico, el carcinoma mucoepidermoide con metaplasia oncocítica y células claras, la variante de células claras del carcinoma epitelial/ mioepitelial y el cistoadenoma papilar linfomatoso (tumor de Whartin).

Su contraparte maligna, el carcinoma oncocítico, es la entidad más importante en el diagnóstico diferencial del oncocitoma. En el primero se producen metástasis en nodos linfoides regionales y a distancia, invasión perineural e intravascular, mitosis, polimorfismo celular y destrucción de tejidos adyacentes (Cao et al., 2015; Westergaard-Nielsen et al., 2018).

Por otro lado, el tumor de Whartin es un tumor con un componente epitelial principalmente oncocítico con una arquitectura quístico-papilar y un estroma linfoide con centros germinativos que lo hacen fácilmente distinguible del oncocitoma (Samar \& Avila, 2017; Sood \& Borah, 2018).

El carcinoma mucoepidermoide es un tumor maligno que frecuentemente demuestra metaplasia oncocítica ycélulas claras. Las células claras de este tumor son PTA/H negativas $\mathrm{y}$, además, la presencia de células mucosas es un requisito para diferenciarlo del oncocitoma (Prabakaran et al.; CocaPelaz et al., 2015).

El oncocitoma y la HOAM con predominio de células claras se deben diferenciar de otras neoplasias con células claras que incluyen el 
adenocarcinoma de células claras, el carcinoma epitelial/ mioepitelial, el carcinoma de células acinares, el mioepitelioma de células claras y el carcinoma de células renales metastásico (Prabakaran et al.).

Las neoplasias con células claras que se desarrollan en las glándulas salivales son en su mayoría malignas a excepción del oncocitoma y el mioepitelioma.

Frecuentemente el adenocarcinoma de células claras presenta un estroma colágeno hialinizado característico, que no se observa en las lesiones oncocíticas. Además, está poco delimitado y su crecimiento es infiltrativo (Prabakaran et al.).

Casos de HOAM tipo células claras han sido erróneamente diagnosticados como adenocarcinoma de células acinares. A diferencia de los oncocitos claros, las células que se encuentran en este último son negativas para glucógeno y PTA/H (Ellis \& Auclair).

El mioepitelioma de células claras se diferencia de las lesiones oncocíticas por presentar exclusivamente mioepiteliocitos PAS positivos que se identifican con marcadores para calponina, caldesmona, miosina de músculo liso de cadena pesada, alfa actina de músculo liso, vimentina, S-100 y p63 (Samar et al., 2014; Samar \& Avila).

El carcinoma epitelial/mioepitelial es un tumor epitelial maligno bifásico de bajo grado. Su estructura histológica consiste en formaciones ductales revestidas por una doble capa de células, interna de epiteliocitos y externa de mioepiteliocitos; su variante de células claras presenta una marcación positiva para p63 y alfa actina de músculo liso. El oncocitoma no muestra diferenciación mioepitelial lo que facilita su diagnóstico (Samar \& Avila; Samar et al., 2018).

Si bien las lesiones metastásicas de la cavidad oral son muy raras, el carcinoma renal de células claras es una neoplasia que puede dar siembras en la región de cabeza y cuello, afectando senos paranasales, lengua, laringe, mandíbula, hueso temporal, tiroides y parótida (Rocca et al., 2017). Cuando compromete la glándula parótida la diferenciación entre tumores a células claras de glándulas salivales y metástasis de cáncer renal resulta difícil con las técnicas histológicas de rutina. Por el contrario, la inmunomarcación para citoqueratinas contribuye al diagnóstico ya que es focalizada en la metástasis renal y difusa en el oncocitoma de glándulas salivales. Otras característi- cas de la metástasis renal, no observadas en nuestras muestras, son su arquitectura heterogénea y la presencia de una red vascular sinusoidal prominente acompañada de hemorragias manifiestas y depósitos de hemosiderina. Además, se ha descrito que p63 es un marcador confiable para diferenciar tumores salivales oncocíticos de metástasis renal (Prabakaran et al.; Avila et al., 2013).

El examen clínico de nuestros pacientes y estudios imagenológicos complementarios corroboraron la ausencia de patología renal detectable.

Se concluye que:

1. Las células eosinofílicas, PTA/H, CK, EMA y anticuerpo antimitocondrial positivas confirman el diagnóstico de patología oncocítica.

2. En tres casos coexisten dos poblaciones celulares de oncocitos, "claros" y "oscuros".

3. Las células claras son oncocitos que acumulan glucógeno que desplaza las mitocondrias hacia la periferia.

4. Su diagnóstico constituye un desafío ya que las características histopatológicas de las lesiones oncocíticas se parecen a otras neoplasias salivales.

AVILA, R. E.; SAMAR, M. E.; FONSECA, I. B.; CORBALL, A. G.; CARRIEL, V.; GARCÍA-MARTINEZ, L. \& RODRÍGUEZ, I. Oncocytic proliferations of salivary glands: structural and immunohistochemical study of 7 cases. Int. J. Odontostomat. 13(1):82-88, 2019.

ABSTRACT: Oncocytes are cells probably originated by metaplastic transformation of the ductal or acinar epithelium of parotid and submandibular. Its proliferation can cause pathological conditions that include multinodular adenomatous oncocytic hiperplasia (HOAM), oncocytomas and oncocytic carcinomas. Oncocytic tumors make up $1 \%$ of all salivary tumors and between 82 and $90 \%$ develop in the parotid; the rest of the tumors are divided between the submandibular gland and the minor salivary glands. Multinodular oncocytic hyperplasias are extremely rare. In the present work we analyzed five cases of parotid oncocytomas and two cases of HOAM, one of parotid and the other of submandibular; structural and immunohistochemical characteristics of the oncocytes were described. Biopsies were included in paraffin, serial cuts were stained with H\&E, Hematoxylin / phosphotungstic acid (PTA / $\mathrm{H}), \mathrm{PAS}$ and were marked with antimitochondrial AC, CK $5 / 6$, CKs 20 and EMA. The tumors showed a nodular growth encapsulated by dense connective tissue. The histological cuts showed dark eosinophilic oncocytes and violaceous granulations with PTA / H. In two cases of parotid and the case of submandibular HOAM, PAS positive clear oncocytes were also present. The immunostaining was positive in all the cells, being the labeling for peripheral mitochondria in the clear oncocytes. 
Eosinophilic cells PTA / $\mathrm{H}$ positive with strongly marked with antimitochondrial AC, CKs and EMA confirm the diagnosis of oncocytic pathology. In three cases, light and dark oncocytes coexist. Clear cells are oncocytes that accumulate glycogen in their cytoplasm, displacing the mitochondria to the periphery. In the differential diagnosis we should consider salivary tumors with clear cells, metastatic renal carcinoma, Whartin's tumor, the clear cell variant of epithelial / myoepithelial carcinoma and mucoepidermoid carcinoma with oncocytic metaplasia.

KEY WORDS: salivary glands, oncocytes, oncocitoma, multinodular adenomatous oncocytic hyperplasia, histology, immunohistochemistry.

\section{REFERENCIAS BIBLIOGRAFICAS}

Alcaraz-Mateos, E.; Vázquez-Olmos, C.; Piqueras-Pérez, F. M.; NovoaJuiz, V.; Pérez-Ramos, M. \& Giménez-Badcuñana, A. Hiperplasia nodular oncocítica multifocal de glándula parótida. A propósito de un caso con revisión del espectro de lesiones oncocíticas. Rev. Esp. Patol., 45(3):169-74, 2012.

Avila, R. E.; Samar, M. E.; Ferraris, L.; Ferraris, R. V.; Fonseca, I, Corball, A.; Asís, O. G. \& Olmedo, L. Non-hyalinizing clear cell carcinoma of the parotid: report of two cases with different grade of differentiation. Int. J. Morphol., 31(3):1056-61, 2013.

Cao, Y.; Zhu, M.; Mao, R.; Cao, R.; Yu, G. \& Niu, A. Oncocytic carcinoma of the salivary gland with thymoma: A case report and review of the literature. Oncol. Lett., 9(2):681-4, 2015.

Çiris, I.M.; Bapinar, S..; Asim Aydin, M.; Aydogan, B. \& Kapucuoglu, N. Multifocal nodular oncocytic hyperplasia of the parotid gland. Erciyes Tip. Dergisi, 33:243-6, 2011.

Coca-Pelaz, A.; Rodrigo, J. P.; Triantafyllou, A.; Hunt, J. L.; Rinaldo, A.; Strojan, P.; Haigentz, M. Jr.; Mendenhall, W. M.; Takes, R. P.; Vander Poorten, V. \& Ferlito, A. Salivary mucoepidermoid carcinoma revisited. Eur. Arch. Otorhinolaryngol., 272(4):799-819, 2015.

El-Naggar, A. K.; Chan, J. K. C.; Grandis, J. R.; Takata, T. \& Slootweg, P. J. (Eds.). Classification of Head and Neck Tumours. Vol. 9. 4th ed. Lyon, World Health Organization, IARC Press, 2017.

Ellis, G. L. \& Auclair, P. L. Tumors of the Salivary Glands, AFIP Atlas of Tumor Pathology Series 4. Washington D. C., AFIP, 2008.

Evren, C.; Demirbilek, N.; Yig it, V. B. \& Kaur, A. C. Oncocytoma of the parotid gland complicated by hypercalcemia: a case report. Kulak Burun Bogaz Ihtis. Derg., 25(3):179-81, 2015.

Kinoshita, Y.; Harada, H.; Kobayashi, T. K.; Yoshizawa, K.; Yuri, T.; Takasu, K.; Tsubura, A. \& Shikata, N. Multifocal adenomatous oncocytic hyperplasia of the parotid gland. Case Rep. Oncol., 7(3):819-24, 2014.

Kontaxis, A.; Zanarotti, U.; Kainz, J. \& Beham, A. Diffuse hyperplastic oncocytosis of the parotid gland. Laryngorhinootologie, 83(3):185-8, 2004

Kumar, R.; Natarajan, S.; Sneha, K. S.; Chitra, N. S.; Boaz, K. \& Manaktala, $\mathrm{N}$. Oncocytes in mucoepidermoid carcinoma of the palate: diagnostic challenges. Case Rep. Dent., 2017:5741821, 2017.

Loreti, A.; Sturla, M.; Gentileschi. S.; Bracaglia. R.; Prat. Y.; Fadda. G. \& Farallo, E. Diffuse hyperplastic oncocytosis of the parotid gland. Br. J. Plast. Surg., 55(2):151-2, 2002.

Mandel, L. \& Carrao, V. Bilateral parotid diffuse hyperplastic oncocytosis: case report. J. Oral Maxillofac. Surg., 63(4):560-2, 2005.

Motallebnejad, M.; Seyedmajidi M.; Khakbaz Baboli, O. \& Yarmand, F. Oncocytoma of palatal minor salivary gland. Arch. Iran. Med., 18(5):320-1, 2015.

Palakshappa, S. G.; Bansal, V.; Reddy, V. \& Kamarthi, N. Oncocytoma of minor salivary gland involving the retromolar region: A rare entity. $\mathrm{J}$. Oral Maxillofac. Pathol., 18(1):127-30, 2014.

Politi, M.; Toro, C.; Cian, R.; Robiony, M. \& Zerman, N. Multifocal adenomatous oncocytic hyperplasia of the parotid gland. Oral. Oncol. Extra, 41(8):183-7, 2005.
Prabakaran, S. S.; Chen, F. \& Aguirre, A. Oncocytoma of the parotid gland and its mimickers: a comprehensive review. North Am. J. Med. Sci., 3(4):171-80, 2010.

Rocca, B. J.; Gini, A.; Calandra, C.; Ginori, A. \& Crivelli, F. Parotid metastasis of renal cell carcinoma, mimicking primary clear cell oncocytoma: report of a case and brief review of the literature. Pathologica, 109(4):384-8, 2017.

Rooper, L. M.; Onenerk, M.; Siddiqui, M. T.; Faquin, W. C.; Bishop, J. A. \& Ali, S. Z. Nodular oncocytic hyperplasia: Can cytomorphology allow for the preoperative diagnosis of a nonneoplastic salivary disease? Cancer Cytopathol., 125(8):627-34, 2017.

Samar, M. E. \& Avila, R. E. Glosario de Histopatología: Tumores Epiteliales de las Glándulas Salivales. $3^{\mathrm{a}}$ ed. Ciudad de Córdoba, Samar Ediciones (autor editor), 2017.

Samar, M. E.; Avila, R. E. \& Esteban Ruiz, F. Técnicas Histológicas. Ciudad de Córdoba, Editorial SeisC, 2004.

Samar, M. E.; Avila, R. E.; Fonseca, I. B.; Anderson, W. J. \& Fonseca, G. M. Clear cell-variant of epithelialmyoepithelial carcinoma of the parotid gland: the role of immunohistochemistry. South Afr. Dent J., 73(3):1603, 2018.

Samar, M. E.; Avila, R. E.; Furnes, M. S.; Fonseca, I. B.; Juri, H. O.; Olmedo, L. A. \& Anderson, W. J. Epithelioid myoepithelioma of the parotid gland: a histopathological and immunohistochemical study. Int. J. Med. Surg. Sci., 1(2):177-83, 2014

Saqi, A.; Giorgadze, T. A.; Eleazar, J.; Remotti, F. \& Vazquez, M. F. Clear cell and eosinophilic oncocytomas of salivary gland: cytological variants or parallels? Diagn. Cytopathol., 35(3):158-63, 2007.

Sepúlveda, I.; Platín, E.; Spencer, M. L.; Mucientes, P.; Frelinghuysen, M.; Ortega, P. \& Ulloa, D. Oncocytoma of the parotid gland: a case report and review of the literature. Case Rep. Oncol., 7(1):109-16, 2014.

Sood, N. \& Borah, P. Warthins tumor: Cyto histological spectrum with emphasis on diagnostic difficulties. Diagn. Cytopathol., 46(7):613-9, 2018.

Strassburger, S.; Hyckel, P. \& Kosmehl, H. Multifocal oncocytic adenomatous hyperplasia of the parotid gland. A case report. Int. J. Oral Maxillofac. Surg., 28(6):457-8, 1999.

Thompson, L. D.; Wenig, B. M. \& Ellis, G. L. Oncocytomas of the submandibular gland. A series of 22 cases and a review of the literature. Cancer, 78(11):2281-7, 1996

Vera-Sampere, F. \& Vera-Sirera, B. Utilidad del anticuerpo antimitocondrial 113-1 en el diagnóstico y categorización de los tumores de glándula salival con diferenciación oncocítica. Acta Otorrinolaringol. Esp., 62(1):1-9, 2011

Vlachaki, E.; Tsapas, A.; Dimitrakopoulos, K.; Kontzoglou, G. \& Klonizakis, I. Parotid gland oncocytoma: a case report. Cases J., 2:6423, 2009

Westergaard-Nielsen, M.; Godballe, C.; Andersen, L. J.; Primdahl, H.; Kristensen, C. A.; Andersen, E. \& Bjørndal, K. Oncocytic carcinoma of the salivary glands: A Danish national study. Aurix Nasus Larynx, 45(4):825-30,2018.

Zagólski, O.; Czajecki, K. \& Gajda, M. Diffuse hyperplastic oncocytosis of the parotid gland. Pol. Merkur. Lekarski., 21:356-7, 2006

Zhou, C. X. \& Gao, Y. Oncocytoma of the salivary glands: a clinicopathologic and immunohistochemical study. Oral Oncol., 45(12):e232-8, 2009.

Dirección para correspondencia:

Rodolfo Esteban Avila

Catamarca 1546. (5000) Córdoba

ARGENTINA

Email: avilainfo@yahoo.com.ar

Recibido : 07-09-2018

Aceptado: 19-10-2018 